\title{
Endovascular arch replacement with an endoprosthesis with three inner branches
}

\author{
Stéphan Haulon ${ }^{1}$, Raphael Soler ${ }^{1,2}$, A. Claire Watkins ${ }^{1,3}$, Philippe Amabile ${ }^{2}$, Elie Fadel ${ }^{1}$, Dominique Fabre ${ }^{1}$ \\ ${ }^{1}$ Aortic Center, Department of Aortic and Vascular Surgery, Hôpital Marie Lannelongue, Université Paris Sud, France; ${ }^{2}$ Vascular Surgery, CHU La \\ Timone, Marseille, France; ${ }^{3}$ Department of Cardiothoracic Surgery, Stanford University School of Medicine, Stanford, CA, USA \\ Correspondence to: Pr. Stéphan Haulon. Aortic Centre, Department of Aortic and Vascular Surgery, Hôpital Marie Lannelongue, Le Plessis-Robinson, \\ INSERM UMR_S 999, Université Paris Sud, France. Email: s.haulon@hml.fr.
}

Submitted Mar 07, 2018. Accepted for publication Mar 23, 2018.

doi: $10.21037 /$ acs.2018.04.07

View this article at: http://dx.doi.org/10.21037/acs.2018.04.07

\section{Clinical vignette}

A 78-year-old female was referred to us with a large aortic arch aneurysm with a maximal diameter of $66 \mathrm{~mm}$. Her past medical history includes chronic obstructive pulmonary disease and a recent thoracoscopic segmentectomy. Due to this history, she was considered at high risk for an open repair.

Standard endovascular repair with a two-branch endograft [one for the brachiocephalic trunk (BCT), one of the left common carotid (LCC)] involves an initial left subclavian artery (LSA) debranching onto the LCC artery, followed a few weeks later by implantation of the branched endograft. The procedure is often staged to avoid excessive bleeding from the cervicotomy that can occur with anticoagulation during the endovascular procedure. To avoid repetitive general anesthesia in this fragile patient, we decided to implant a 3-branch endograft, manufactured by Cook Medical (Bloomington, IN) with two antegrade branches for the BCT and the LCC and one retrograde inner branch for the LSA. A preloaded catheter was positioned into the LSA branch to directly access it from the femoral access. The branched arch device is currently not approved by the U.S. Food and Drug Administration.

\section{Surgical techniques}

The procedure was performed in a latest-generation hybrid room (Discovery ${ }^{\mathrm{TM}}$ IGS 740, GE Healthcare, Chalfont, UK) under general anesthesia. An arterial line was positioned in the right radial artery and a center line in the right jugular vein.

Because the delivery system is loaded into a 22-French sheath, we first performed a conduit to the right common iliac artery with a right retroperitoneal approach. The $10-\mathrm{mm}$ Dacron graft was sutured distally to the right common femoral artery. A 6-French right and left common carotid access were performed to catheterize the BCT and LCC side branches. Covered bridging stents were inserted through this access and deployed within inner side branches of the arch device.

After systemic heparinization with $100 \mathrm{IU} / \mathrm{kg}$ [target: activated clotting time (ACT) $>300 \mathrm{~s}$ ], catheters and/or sheaths were placed at the origins of the BCT and LCC. A pigtail catheter was positioned into the apex of the left ventricle from the conduit access and a stiff, curved wire (Lunderquist $^{\circledR}$ Extra-Stiff Wire Guide, Cook Medical, Bloomington, IN, USA) was advanced through this catheter. The position of the tip of the stiff wire was constantly visualized. Under fluoroscopy, the orientation of the main body of the graft is verified outside the patient, then delivered over the stiff wire to the aortic arch. The tapered short tip was brought through the aortic valve into the left ventricle. An angiogram was performed, demonstrating the branches, with their associated markers positioned adequately, and the graft was deployed under rapid pacing.

Normal cardiac output was resumed prior to withdrawing the tapered tip of the delivery system and the stiff wire from the left ventricle. The side branches on the aortic device are catheterized from the target vessels and sheaths are positioned into the inner side-branches. After deployment of the BCT (Cook Medical, Bloomington, IN, USA) and LCC (Fluency ${ }^{\circledR}$, Bard, Tempe, AZ, USA) bridging stents, a second Lunderquist guide wire is advanced into 
the LSA branch preloaded catheter. The delivery system of the aortic endograft and its guide wire were retrieved. A 22-French, 30-cm sheath was advanced over the LSA branch wire. A 12-French, $80-\mathrm{cm}$ sheath $\left(\right.$ Flexor $^{\circledR}$, Cook medical) was then advanced through the 22-French sheath, over the guide wire, directly into the LSA branch. The valve of this 12 -French sheath was punctured to advance a second floppy wire and a $100 \mathrm{~cm}$ angled catheter, which were used to catheterize the LSA. Once the catheter had been advanced into the left axillary artery, the floppy wire was replaced by a stiffer wire (Amplatz). The Lunderquist wire was then retrieved and a bridging self-expandable, covered stent advanced (Viabahn, WL Gore, Flagstaff, AZ, USA) over the guidewire. It was sized and positioned to completely overlap the inner branch on one side and to provide maximum overlap in the LSA without covering the origin of the left vertebral artery on the other side. It was relined with a nitinol, self-expandable stent to provide more radial force and a more 'kink-resistant' branch.

The completion angiogram and non-contrast cone beam CT study confirmed technical success. The patient stayed one day in the ICU. She was discharged home on postoperative day 7. No stroke, cardiac or renal events occurred during the peri-operative period. All branches were patent on the postoperative CT and duplex ultrasound.

\section{Comment}

We describe a totally endovascular aortic arch aneurysm repair, using an endovascular arch branched graft with three inner branches. There are many advantages to the techniques described here, including simplification of branch catheterization, cerebral protection and physiologic burden to the patient. Including a third branch avoids revascularisation of the LSA during a prior or same-day procedure. This serves to reduce the complexity of the procedure and avoid potential complications, including nervous or lymphatic injury.

During the procedure two small, $2-\mathrm{cm}$ carotid cutdowns were performed, allowing carotid clamping (and thus brain protection) while inserting and implanting the bridging stents in the BCT and LCC. This retrograde access path to the inner branches is straight and without tortuosity. We find the BCT and LCC side branches easier to cannulate from above rather than from the subclavian arteries, potentially reducing the overall procedural time. Additionally, the retrograde, rather than antegrade, design of the LSA branch provides more working space in the arch lumen and can accommodate future intervention of the visceral aorta from a cranial approach.

One of the benefits of the delivery system of the arch branch device is the ease of the use of the system, especially the LSA branch preloaded system. It does not require snaring a wire in the arch or tracking a delivery system over two wires. Reducing endovascular manoeuvers in the arch is of paramount importance to reduce the stroke risk. We consider this to be the greatest advantage of the techniques described here.

In this case, blood flow through side branches in the aortic device provided adequate cerebral perfusion prior to completion of each side branch. Very brief, separate occlusion of each carotid artery during stent deployment allowed embolic protection. This cerebral perfusion strategy was safe in our elderly patient and is significantly simplified compared to other endovascular aortic arch procedures.

Potential disadvantages of a totally endovascular arch repair include crowding of side branches within the arch, leading to a pressure gradient between the ascending and descending aorta. This can be avoided with careful imaging review and procedural planning.

It remains unclear whether total endovascular therapy of aortic arch disease may significantly reduce risk compared to open or hybrid techniques. The variety of surgical approaches and the reservation of endovascular therapy for high-risk, often inoperable patients, muddies direct comparison of outcomes. Regardless of surgical approach, endovascular aortic arch repair remains a morbid procedure, with recent reviews documenting 3-12\% early mortality, $5-15 \%$ stroke, $1-6 \%$ paraplegia and $1-7 \%$ retrograde dissection risk (1-3). Fine-tuning of endovascular devices and techniques, will be critical to improve outcomes in the evolving field of endovascular aortic arch surgery. This case highlights nuanced endovascular methods facilitating swift endovascular aortic arch repair.

In conclusion, we describe the successful, totally endovascular, three-branched aortic arch repair using a combined antegrade and retrograde side branch approach, without early complication.

\section{Acknowledgements}

None.

\section{Footnote}

Conflicts of Interest: S Haulon is a consultant for Cook 
Medical and GE Healthcare. The other authors have no conflicts of interest to declare.

\section{References}

1. Rudarakanchana N, Jenkins MP. Hybrid and total endovascular repair of the aortic arch. Br J Surg 2018;105:315-27.

Cite this article as: Haulon S, Soler R, Watkins AC, Amabile P, Fadel E, Fabre D. Endovascular arch replacement with an endoprosthesis with three inner branches. Ann Cardiothorac Surg 2018;7(3):431-433. doi: 10.21037/acs.2018.04.07
2. Clough RE, Spear R, Van Calster K, et al. Case series of aortic arch disease treated with branched stent-grafts. Br J Surg 2018;105:358-65.

3. Spanos K, Tsilimparis N, Rohlffs F, et al. Total endovascular arch repair is the procedure of the future. Part I. J Cardiovasc Surg (Torino) 2018. [Epub ahead of print]. 